\title{
CONVEX AND STARLIKE MEROMORPHIC FUNCTIONS
}

\author{
JAMES MILLER
}

\begin{abstract}
In this paper we study meromorphic univalent functions with a simple pole at $p$ which map the unit disk onto the exterior of convex sets or the exterior of starlike sets. A question raised by Libera and Livingston concerning the equality of two classes of starlike meromorphic univalent functions is answered. We show that the set of points omitted by the class of convex functions is $\left\{-p /\left(1+p^{2}\right)\right\}$. The coefficients of convex and starlike meromorphic functions are also studied.
\end{abstract}

1. Introduction. Let $S(p), 0<p<1$, denote the class of univalent meromorphic functions $f$ in the unit disk $E$ with a simple pole at $z=p$ and with the normalization $f(z)=z+a_{2} z^{2}+\cdots$ for $|z|<p$. Let $K(p)$ be the subclass of $S(p)$ defined by $f \in K(p)$ if and only if $f \in S(p)$ and there is a $\rho, p<\rho<1$, such that for each $z, \rho<|z|<1$,

$$
\operatorname{Re}\left\{1+z f^{\prime \prime}(z) / f^{\prime}(z)\right\}<0 .
$$

Functions in $K(p)$ map $|z|<r, r>\rho$ (for some $\rho, p<\rho<1$ ), onto the complement of a convex set. Further, if $f \in K(p)$, then for each $z \in E$

$$
\operatorname{Re}\left\{1+z \frac{f^{\prime \prime}(z)}{f^{\prime}(z)}+\frac{2 p}{z-p}-\frac{2 p z}{1-p z}\right\}<0 .
$$

Define $\Sigma(p)$ to be the functions $f \in S(p)$ which satisfy (1). Functions in $\Sigma(p)$ map $E$ onto the exterior of a convex set [4]. For $p<2-\sqrt{3}$ Royster [5] showed that $K(p)=\Sigma(p)$ and for $p>2-\sqrt{3}$ that $K(p)$ is a proper subset of $\Sigma(p)$.

Also let $S^{*}\left(p, w_{0}\right)$ denote the subclass of $S(p)$ defined by $f \in S^{*}\left(p, w_{0}\right)$ if and only if $f \in S(p)$ and there is a $\rho, p<\rho<1$, such that for each $z, \rho<|z|<1$,

$$
\operatorname{Re}\left\{\frac{z f^{\prime}(z)}{f(z)-w_{0}}\right\}<0 .
$$

If $f \in S^{*}\left(p, w_{0}\right)$, then

$$
\operatorname{Re}\left\{\frac{z f^{\prime}(z)}{f(z)-w_{0}}+\frac{p}{z-p}-\frac{p z}{1-p z}\right\}<0
$$

for $z \in E$. Now define $\Sigma^{*}\left(p, w_{0}\right)$ to be the functions $f \in S(p)$ which satisfy (2).

2. Starlike functions. In [3] it was shown that $S^{*}\left(p, w_{0}\right)=\Sigma^{*}\left(p, w_{0}\right)$ for $p<2-\sqrt{3}$. We now show that this is true for $p<\sqrt{3-2 \sqrt{2}}$, and that this is best possible for a certain $w_{0}$.

Received by the editors November 4, 1979 and, in revised form, January 8, 1980.

AMS (MOS) subject classifications (1970). Primary 30A26, 30A32, 30A34. 
THEOREM 1. If $p<\sqrt{3-2 \sqrt{2}}$ then

$$
S^{*}\left(p, w_{0}\right)=\Sigma^{*}\left(p, w_{0}\right) \text {. }
$$

If $p>\sqrt{3-2 \sqrt{2}}$, then $S^{*}(p, \sigma)$ is a proper subset of $\Sigma^{*}(p, \sigma)$ where

$$
\sigma=-p /\left(1+2 p e^{-i \alpha}+p^{2}\right)
$$

and

$$
\cos \alpha=\frac{2 p^{2}\left(1+r^{2}\right)-\left(1-p^{2}\right) \sqrt{\left(r^{2}-p^{2}\right)\left(1-r^{2} p^{2}\right)}}{2 p r\left(1+p^{2}\right)}
$$

Proof. If $f \in \Sigma^{*}\left(p, w_{0}\right)$, then

$$
\operatorname{Re}\left\{\frac{z f^{\prime}(z)}{f(z)-w_{0}}\right\}=\operatorname{Re}\left\{\frac{p z}{1-p z}-\frac{p}{z-p}\right\}-\operatorname{Re}\{P(z)\}
$$

where $P(0)=1$ and $\operatorname{Re}\{P(z)\}>0$ for $z \in E$. The proof of Theorem 1 in [2] shows that if $p<\sqrt{3-2 \sqrt{2}}$ there exists a $\rho, p<\rho<1$, such that

$$
\operatorname{Re}\left\{\frac{z f^{\prime}(z)}{f(z)-w_{0}}\right\}<0
$$

for $\rho<|z|<1$. Hence $S^{*}\left(p, w_{0}\right)=\Sigma^{*}\left(p, w_{0}\right)$ for $p<\sqrt{3-2 \sqrt{2}}$. Royster [5] has shown that $\operatorname{Re}\{p z /(1-p z)-p /(z-p)\}$ attains its maximum on $|z|=r$, $2-\sqrt{3}<p<r<1$, at $z=r e^{i \alpha}$ where

$$
\cos \alpha=\frac{2 p^{2}\left(1+r^{2}\right)-\left(1-p^{2}\right) \sqrt{\left(r^{2}-p^{2}\right)\left(1-r^{2} p^{2}\right)}}{2 p r\left(1+p^{2}\right)} .
$$

A calculation shows that the function

$$
F(z)=\frac{p z+p \sigma\left(1-e^{-i 2 \alpha}\right) z^{2}}{(p-z)(1-p z)},
$$

where $\sigma=-p /\left(1+2 p e^{-i \alpha}+p^{2}\right)$, belongs to $\Sigma^{*}(p, \sigma)$ for all $p$, but $F \notin S^{*}(p, \sigma)$ for $p>\sqrt{3-2 \sqrt{2}}$.

Recently Libera and Livingston [2] have defined the classes $\Lambda(p)$ and $\Lambda^{*}(p)$ of weakly starlike meromorphic univalent functions with a simple pole at $p$. A function $g$ in $\Lambda(p)$ or $\Lambda^{*}(p)$ omits the origin and $g(0)=1$. If $g \in \Lambda(p)\left(\Lambda^{*}(p)\right.$ resp.), then $f(z)=(g(z)-1) / g^{\prime}(0) \in S^{*}\left(p,-1 / g^{\prime}(0)\right)\left(\Sigma^{*}\left(p,-1 / g^{\prime}(0)\right)\right.$ resp. $)$, and if $f \in S^{*}\left(p, w_{0}\right)\left(\Sigma^{*}\left(p, w_{0}\right)\right.$ resp. $)$, then $g(z)=1-f(z) / w_{0} \in \Lambda(p)\left(\Lambda^{*}(p)\right.$ resp. $)$.

Further, $(1-p)^{2} / p<\left|w_{0}\right|^{-1}<(1+p)^{2} / p$, which are the bounds for $g^{\prime}(0)$. In [2] it was shown that $\Lambda(p)=\Lambda^{*}(p)$ for $p<\sqrt{3-2 \sqrt{2}}$ and that $\Lambda(p)$ is a proper subset of $\Lambda^{*}(p)$ for $p>1 / 2$. Using Theorem 1 we obtain the following result.

COROLlaRy 1. If $p>\sqrt{3-2 \sqrt{2}}$, then $\Lambda(p)$ is a proper subset of $\Lambda^{*}(p)$.

Remark. In Theorem 1 we have not shown that $S^{*}\left(p, w_{0}\right)$ is a proper subset of $\Sigma^{*}\left(p, w_{0}\right)$ for all possible $w_{0}$ and $p>\sqrt{3-2 \sqrt{2}}$. Furthermore, since $\sigma$ depends on $\alpha$ we feel that this is unlikely to be true. 
3. Convex functions. Sheil-Small [6] and Suffridge [7] have shown that $f(z)=a_{1} z$ $+a_{2} z^{2}+\cdots$ is convex in $E$ if and only if

$$
\operatorname{Re}\left\{\frac{2 z f^{\prime}(z)}{f(z)-f(\xi)}-\frac{z+\xi}{z-\xi}\right\}>0, \quad|z|<1,|\xi|<1 .
$$

We now generalize this to functions in $K(p)$ and $\Sigma(p)$.

THEOREM 2. If $f \in K(p)$, then

$$
\operatorname{Re}\left\{\frac{2 z f^{\prime}(z)}{f(z)-f(\xi)}-\frac{z+\xi}{z-\xi}+\frac{z+p}{z-p}-\frac{1+p z}{1-p z}\right\}<0
$$

for $|z|<1$ and $|\xi|<1$.

Proof. For $f \in S(p)$, define $F(z, \xi)$, for $|z|<1$ and $|\xi|<1$, by

$$
F(z, \xi)=\left\{\begin{array}{l}
1+\frac{z f^{\prime \prime}(z)}{f^{\prime}(z)}+\frac{z+p}{z-p}-\frac{1+p z}{1-p z}, \text { if } z=\xi, \\
\frac{2 z f^{\prime}(z)}{f(z)-f(\xi)}-\frac{z+\xi}{z-\xi}+\frac{z+p}{z-p}-\frac{1+p z}{1-p z}, \quad \text { if } z \neq \xi .
\end{array}\right.
$$

Then $F(z, \xi)$ is analytic in $E \times E$ for each $f \in S(p)$. Now suppose $f \in K(p)$. Let $\rho(f)$ be such that if $\rho(f)<|z|<1$, then

$$
\operatorname{Re}\left\{1+z f^{\prime \prime}(z) / f^{\prime}(z)\right\}<0 .
$$

For $0<r<1$, let $C(r)=\{f(z)|:| z \mid=r\}$. Then $C(r)$ is a convex curve for $r>\rho(f)$. This says that $\arg \left\{f\left(r e^{i t}\right)-f\left(r e^{i \theta}\right)\right\}$ is a decreasing function of $t$, for $t \in$ $(\theta, \theta+2 \pi)$. Therefore

$$
\operatorname{Re}\left\{\frac{z f^{\prime}(z)}{f(z)-f(\xi)}\right\}=\frac{\partial}{\partial t} \arg \left\{f\left(r e^{i t}\right)-f\left(r e^{i \theta}\right)\right\}<0
$$

for $z=r e^{i t} \neq \xi=r e^{i \theta}$. Further if $|z|=|\xi|$ and $z \neq \xi$ then

$$
\operatorname{Re}\left\{\frac{z+\xi}{z-\xi}\right\}=0 \text {. }
$$

Thus

$$
\operatorname{Re}\left\{\frac{2 z f^{\prime}(z)}{f(z)-f(\xi)}-\frac{z+\xi}{z-\xi}\right\}<0
$$

for $|z|=|\xi|>\rho(f)$ and $z \neq \xi$. Since $F(z, \xi)$ is analytic for all $z$ and $\xi(|z|<1$ and $|\xi|<1)$ and

$$
\operatorname{Re}\left\{\frac{z+p}{z-p}-\frac{1+p z}{1-p z}\right\}=0
$$

for $|z|=1$, the maximum principle leads to (3).

THEOREM 3. Let $f \in S(p)$. Then $f \in \Sigma(p)$ if and only if

$$
\operatorname{Re}\left\{\frac{2 z f^{\prime}(z)}{f(z)-f(\xi)}-\frac{z+\xi}{z-\xi}+\frac{z+p}{z-p}-\frac{1+p z}{1-p z}\right\}<0
$$

for $|z|<1$ and $|\xi|<1$. 
Proof. Let $F(z, \xi)$ be as defined in (4). Then

$$
F(z, z)=1+\frac{z f^{\prime \prime}(z)}{f^{\prime}(z)}+\frac{z+p}{z-p}-\frac{1+p z}{1-p z} .
$$

Hence (5) implies that $f \in \Sigma(p)$.

If $f \in \Sigma(p)$, then by combining the proof of Theorem 2 and Carathéodory's theorem on variable regions we have (5).

Corollary 2. If $f \in \Sigma(p)$, then

$$
\operatorname{Re}\left\{\frac{z f^{\prime}(z)}{f(z)}+\frac{p}{z-p}-\frac{p z}{1-p z}\right\}<\frac{1}{2} .
$$

Proof. Let $\xi=0$ in (5).

For $f \in \Sigma(p)$ Theorem 3 says

$$
\frac{2 z f^{\prime}(z)}{f(z)-f(\xi)}-\frac{z+\xi}{z-\xi}+\frac{z+p}{z-p}-\frac{1+p z}{1-p z}=-P(z)
$$

where $P(0)=1$ and $\operatorname{Re} P(z)>0$ for $|z|<1$. Thus for $\xi \neq 0$ we have

$$
P(z)=1+\left(\frac{1}{f(\xi)}-\frac{1}{\xi}+\frac{1}{p}+p\right) 2 z+\cdots
$$

From the bound on the coefficient of $z$, we have

THEOREM 4. If $f \in \Sigma(p)$, then

$$
\left|\frac{1}{f(z)}-\frac{1}{z}+\frac{1}{p}+p\right|<1 .
$$

THEOREM 5. For the class $\Sigma(p)$, we have

$$
\left\{\frac{-p}{\left(1+p^{2}\right)}\right\}=\bigcap_{f \in \Sigma(p)}\{C / f(E)\}
$$

Proof. The functions

$$
f_{\lambda}(z)=\frac{p z-p^{2}(1+\lambda) z^{2} /\left(1+p^{2}\right)}{p-\left(1+p^{2}\right) z+p z^{2}}, \quad|\lambda|=1,
$$

map $E$ onto the exterior of straight line segments through $w=-p /\left(1+p^{2}\right)$. Thus $-p /\left(1+p^{2}\right)$ is the only possible point in the exterior of $f(E)$ for all $f \in \Sigma(p)$. Suppose $f(z)=-p /\left(1+p^{2}\right)$ for some $z$. Then (7) gives $|-1 / z|<1$, but we have $|z|<1$.

Since functions in $\Sigma(p)$ omit $-p /\left(1+p^{2}\right)$ and map $E$ onto the exterior of a convex set, we obtain the following.

TheOREM 6. If $f \in \Sigma(p)$, then $f \in \Sigma^{*}\left(p,-p /\left(1+p^{2}\right)\right)$.

TheOREM 7. If $f \in K(p)$, then $f \in S^{*}\left(p,-p /\left(1+p^{2}\right)\right)$. 
4. Coefficient bounds. Let $\Sigma^{*}$ denote the class of functions $g(z)=1 / z+c_{0}+$ $c_{1} z+\cdots$, which are starlike in $0<|z|<1$.

If $f \in \Sigma^{*}\left(p, w_{0}\right)$, then the function

$$
g(z)=\frac{(z-p)(1-p z)}{p z w_{0}}\left(f(z)-w_{0}\right)
$$

is in $\Sigma^{*}$, since

$$
\operatorname{Re} \frac{z g^{\prime}(z)}{g(z)}=\operatorname{Re}\left\{\frac{z f^{\prime}(z)}{f(z)-w_{0}}+\frac{p}{z-p}-\frac{p z}{1-p z}\right\}<0
$$

for $|z|<1$. Further if $g \in \Sigma^{*}$ then

$$
f(z)=w_{0}\left(1-\frac{p z}{(p-z)(1-p z)} g(z)\right)
$$

is in $\Sigma^{*}\left(p, w_{0}\right)$, for $-w_{0}=\left(b_{0}+p+1 / p\right)^{-1}$ where $g(z)=1 / z+b_{0}+b_{1} z+\cdots$.

Taking the series expansions in (8) with $f(z)=z+a_{2} z^{2}+\cdots, g(z)=1 / z+$ $b_{0}+b_{1} z+\cdots$, and $w_{0}=-\left(b_{0}+p+1 / p\right)^{-1}$, we have

$$
\begin{aligned}
g(z)= & \frac{1}{z}-\left(p+\frac{1}{p}+\frac{1}{w_{0}}\right)+\left(1+\frac{1+p^{2}}{p w_{0}}-\frac{a_{2}}{w_{0}}\right)^{z} \\
& +\cdots-\frac{1}{w_{0}}\left(a_{n-1}-a_{n} \frac{\left(1+p^{2}\right)}{p}+a_{n+1}\right) z^{n}+\cdots
\end{aligned}
$$

Using the bound $\left|b_{n}\right|<2(n+1)^{-1}[1]$, we have the following result.

THEOREM 8. If $f \in \Sigma^{*}\left(p, w_{0}\right)$, then

$$
\left|a_{2}-\frac{1+p^{2}}{p}-w_{0}\right|<\left|w_{0}\right|
$$

and

$$
\left|a_{n-1}-\frac{1+p^{2}}{p} a_{n}+a_{n+1}\right|<\frac{2\left|w_{0}\right|}{n+1} \text { for } n>2
$$

Equality occurs in (9) for the functions

$$
f_{\lambda}(z)=\frac{p z+p w_{0}(1+\lambda) z^{2}}{(p-z)(1-p z)} \quad \text { where }|\lambda|=1 \text {. }
$$

Since $\Sigma(p) \subset \Sigma^{*}\left(p,-p /\left(1+p^{2}\right)\right)$, we have

THEOREM 9. If $f \in \Sigma(p)$, then

$$
\left|a_{2}-\frac{1+p^{2}+p^{4}}{p\left(1+p^{2}\right)}\right|<\frac{p}{1+p^{2}}
$$

and

$$
\frac{1+p^{4}}{p\left(1+p^{2}\right)}<\left|a_{2}\right|<\frac{1+p^{2}}{p}
$$


Equality occurs in (11) for

$$
f_{\lambda}(z)=\frac{p z-p^{2}(1+\lambda) z^{2} /\left(1+p^{2}\right)}{(p-z)(1-p z)} \text { for }|\lambda|=1 \text {. }
$$

Equality in (12) occurs for $f_{1}$ and $f_{-1}$.

Royster obtained (12) for $0<p<2-3$. Here we obtained the second coefficient region for all $p$.

Corollary 3. If $f \in \Sigma^{*}\left(p, w_{0}\right)$, then

$$
\frac{R(p)}{3 p^{2}(1-p)^{2}}<\operatorname{Re} a_{3}<\frac{1+p^{2}+p^{4}}{p^{2}}
$$

where $R(p)=3-6 p-8 p^{3}-6 p^{3}-6 p^{5}+3 p^{6}$. If $p_{0} \simeq 0.4037$ is the smallest positive root of $R(p)$ and $p<p_{0}$, then $\operatorname{Re} a_{3}>0$.

Proof. The right side of the inequality follows from the fact that

$$
\left|a_{3}\right|<\left(1+p^{2}+p^{4}\right) / p^{2}
$$

for all functions in $S(p)$. From (9) we see that $a_{2}=\left(1+p^{2}\right) / p+w_{0}(1+\lambda)$ where $|\lambda|<1$. Using this for $a_{2}$ and equation (10), we have

$$
\left|1-\frac{\left(1+p^{2}\right)^{2}}{p^{2}}-\frac{1+p^{2}}{p} w_{0}(1+\lambda)+a_{3}\right|<\frac{2\left|w_{0}\right|}{n+1}
$$

and then

$$
\left|a_{3}-\frac{1+p^{2}+p^{4}}{p^{2}}\right|<\left(\frac{2}{3}+\frac{2\left(1+p^{2}\right)}{p}\right)\left|w_{0}\right|<\frac{\left(6+2 p+6 p^{2}\right)}{3(1-p)^{2}}
$$

Thus we have

$$
\frac{3-6 p-8 p^{3}-6 p^{5}+3 p^{6}}{3 p^{2}(1-p)^{2}}<\operatorname{Re} a_{3}
$$

Corollary 4. If $f \in \Sigma(p)$, then

$$
\left|a_{3}-\frac{p^{2}+1}{p} a_{2}+1\right|<\frac{2 p}{3\left(1+p^{2}\right)}
$$

and

$$
\operatorname{Re} a_{3}>\frac{3-2 p^{3}+3 p^{6}}{3 p^{2}\left(1+p^{2}\right)}>\frac{2}{3}
$$

Proof. Since $F \in \Sigma^{*}\left(p,-p /\left(1+p^{2}\right)\right)$, we have

$$
a_{2}=\frac{1+p^{2}+p^{4}}{p\left(1+p^{2}\right)}+\frac{p \lambda}{1+p^{2}}, \quad|\lambda|<1,
$$


and

$$
\left|a_{3}-\frac{1+p^{2}}{p} a_{2}+1\right|<\frac{2}{3} \frac{p}{1+p^{2}}
$$

Thus

$$
\left|a_{3}-\frac{1+p^{4}}{p^{2}}-\lambda\right|<\frac{2}{3} \frac{p}{1+p^{2}},
$$

or

$$
\operatorname{Re} a_{3}>\frac{3-2 p^{3}+3 p^{6}}{3 p^{2}\left(1+p^{2}\right)}>\frac{2}{3} \text {. }
$$

\section{REFERENCES}

1. J. Clunie, On meromorphic schlicht functions, J. London Math. Soc. 34 (1959), 215-216. MR 21 \#5737.

2. R. J. Libera and A. E. Livingston, Weakly starlike meromorphic univalent functions, Trans. Amer. Math. Soc. 202 (1975), 181-191. MR 50 \# 13482.

3. J. Miller, Convex meromorphic mappings and related functions, Proc. Amer. Math. Soc. 25 (1970), 220-228. MR 41 \# 3740.

4. J. A. Pfaltzgraff and B. Pinchuk, A variational method for classes of meromorphic functions, J. Analyses Math. 24 (1971), 101-150. MR 43 \# 7613.

5. W. C. Royster, Convex meromorphic functions, Mathematical Essays Dedicated to A. J. Macintyre, Ohio Univ. Press, Athens, Ohio, 1970, pp. 331-339. MR 42 \#7883.

6. T. Sheil-Small, On convex univalent functions, J. London Math. Soc. (2) 1 (1969), 483-492. MR 40 \#5844.

7. T. J. Suffridge, Some remarks on convex maps of the unit disk, Duke Math. J. 37 (1970), 775-777. MR 42 \#4722.

Department of Mathematics, West Virginia University, Morgantown, West Virgnia 26506 